\title{
INVESTIGACIÓN
}

Recibido: 02/02/2018 --- Aceptado: 18/04/2018 --- Publicado: 15/09/2018

\section{INVESTIGACIÓN CUALITATIVA: FUNDAMENTOS EPISTEMOLÓGICOS, TEÓRICOS Y METODOLÓGICOS}

\section{Qualitative research: epistemological, theoretical and methodological foundations}

José Luis Corona Lisboa. Universidad Nacional Experimental Francisco de Miranda.

Venezuela

joseluiscoronalisboa@gmail.com

\section{RESUMEN}

La investigación cualitativa surge como un paradigma emergente y novedoso, el cual ha recibido la influencia de diversas corrientes filosóficas representadas por: Edgar Morín, Paulo Freire, Edmund Husserl, Martin Heidegger y Schütz Alfred. El objetivo el presente documento fue analizar los fundamentos epistemológicos, teóricos y metodológicos de la investigación cualitativa o interpretativa. La metodología usada fue la investigación documental. Para ello, se realizó una discriminación de artículos científicos indexados en diversos repositorios electrónicos, que brindaron una visión amplia sobre la temática de estudio, en base a diversos autores y la opinión propia del investigador. Se concluye que la investigación cualitativa es un corpus teórico emergente que sustenta su visión epistemológica y metodológica en las experiencias subjetivas e intersubjetivas de los sujetos, cuya práctica se orienta hacia la sociedad construida por el hombre, donde interactúan las versiones y opiniones de los distintos actores sociales, para construir su propia realidad de manera cooperativa y dinámica.

\section{PALABRAS CLAVES}

Investigación cualitativa; paradigma; corrientes filosóficas; epistemología, metodología.

\begin{abstract}
Qualitative research emerges as an emergent and novel paradigm, which has received the influence of diverse philosophical currents represented by: Edgar Morin, Paulo Freire, Edmund Husserl, Martin Heidegger and Schütz Alfred. The objective of this document was to analyze the epistemological, theoretical and methodological foundations of qualitative or interpretative research. The methodology used was documentary research. For this, a discrimination of scientific articles indexed in various electronic repositories was conducted, which provided a broad view on the subject of study, based on various authors and the own opinion of the researcher. It is concluded
\end{abstract}


that qualitative research is an emergent theoretical corpus that supports its epistemological and methodological vision in the subjective and intersubjective experiences of the subjects, the practice of which is oriented towards the society constructed by man, where the versions and opinions of the different social actors interact to build their own reality in a cooperative and dynamic way.

\section{KEY WORDS}

Qualitative research; paradigm; philosophical currents; epistemology; methodology

\section{INVESTIGAÇÃO QUALITATIVA: FUNDAMENTOS EPISTEMOLÓGICOS TEÓRICOS E METODOLÓGICOS}

\section{RESUME}

A investigação qualitativa surge como um paradigma emergente e novo, no qual recebeu influência de diversas correntes filosóficas representadas por Edgar Morín, Paulo Freire, Edmund Alfred. O objetivo do presente documento foi analisar os fundamentos epistemológicos, teóricos e metodológicos da investigação qualitativa ou interpretativa. A metodologia usada foi a investigação documental. Para isso, se realizou uma discriminação de artigos científicos indexados em diversos repertórios eletrônicos, que brindaram uma visão ampla sobre a temática de estudo, em base a diversos autores e a opinião própria do investigador. Se conclui que a investigação qualitativa é um corpus teórico emergente que sustenta sua visão epistemológica e metodológica nas experiências subjetivas e intersubjetivas dos sujeitos, cuja pratica orienta-se a sociedade construída pelo homem, onde interatuam as versões e opiniões dos distintos atores sociais, para construir sua própria realidade de maneira cooperativa e dinâmica.

\section{PALAVRAS CHAVE}

Investigação qualitativa; Paradigma; Correntes filosóficas; Epistemologia; Metodologia

\section{Cómo citar el artículo}

Corona Lisboa, J. L. (2018). Investigación cualitativa: fundamentos epistemológicos, teóricos y metodológicos. [Qualitative research: epistemological, theoretical and methodological foundations] Vivat Academia. Revista de Comunicación, $\mathrm{n}^{\mathrm{o}}$ 144, 69-76. doi: http://doi.org/10.15178/va.2018.144.69-76. Recuperado de http:/ / www.vivatacademia.net/index.php/vivat/article/view/1087

\section{INTRODUCCIÓN}

La Epistemología, es una de las pocas ciencias que le ha permitido al hombre la búsqueda y comprensión del mundo circundante y la relación sujeto-objeto desde distintas perspectivas, para generar, interpretar y transmitir nuevo conocimiento. Entre las diversas corrientes epistemológicas se encuentra el Paradigma Cualitativo o

Vivat Academia. Revista de Comunicación. 15 septiembre/15 diciembre, 2018, n $144,69-76$ 


\section{Corona Lisboa, J. L. Investigación cualitativa: fundamentos epistemológicos, teóricos y metodológicos}

Interpretativo. Entendiéndose por paradigma, aquellas interrelaciones de teorías científicas universalmente aceptadas, que durante cierto tiempo, proporcionan modelos de problemas y soluciones a una comunidad científica en determinada área del saber (Corona, 2016a).

Esta perspectiva filosófica concebida como una metodología de la investigación, es una nueva manera de ver el mundo ya que aporta de forma significativa una experiencia subjetiva de los hechos en su estado natural, tal como son percibidos, estableciéndose la forma de lograr y alcanzar el conocimiento (Sánchez, 2015).

El objetivo del presente documento, fue analizar la crisis de paradigmas y el paradigma emergente desde la modernidad y la postmodernidad, enfatizando los fundamentos epistemológicos, teóricos, metodológicos y axiológicos de la investigación cualitativa o interpretativa, así como, los aportes de los filósofos: Edgar Morín, Paulo Freire, Edmund Husserl, Martin Heidegger y Schütz Alfred en el surgimiento de este paradigma.

\section{METODOLOGÍA}

La metodología usada fue la investigación documental exhaustiva, la cual consiste en revisar material bibliográfico sobre el objeto de estudio para realizar un análisis sobre el contenido seleccionado (Corona, 2016b). Para ello, se realizó una discriminación de artículos científicos indexados en diversos repositorios electrónicos, tesis de postgrado y documentos institucionales, que brindaron una visión amplia sobre la temática de estudio, en base a diversos autores y la opinión propia del investigador.

\section{DESARROLLO}

Para el desarrollo del manuscrito, se tomó en cuenta cuatro puntos claves: fundamentos epistemológicos y metodológicos de la investigación cualitativa, fundamentos teóricos-conceptuales distintivos de la investigación cualitativa (concepto, características y aplicación), filósofos y precursores de la investigación cualitativa. A continuación, se detallan cada uno de los aspectos antes mencionados:

\subsection{Fundamentos Epistemológicos y Metodológicos de la Investigación Cualitativa}

Desde el punto de vista epistemológico, el conocimiento es el constructo teórico interpretativo y subjetivo que el hombre (sujeto cognoscente) da a través de la observación inicial y la interacción recíproca con el objeto de estudio, regido por distintos patrones o estilos de pensamiento determinados, ya sea, a través de su experiencia, de la razón o las vivencias. En el caso de la investigación cualitativa, la obtención del conocimiento viene dado por un estilo de pensamiento vivencialista y se abre paso para la comprensión profunda de los fenómenos sociales a través de la intuición y la conciencia del yo interno, es decir, la conexión entre la conciencia, sentimientos y el ser (Hernández y Padrón, 1997).

Vivat Academia. Revista de Comunicación. 15 septiembre/15 diciembre, 2018, n $144,69-76$ 


\section{Corona Lisboa, J. L. Investigación cualitativa: fundamentos epistemológicos, teóricos y metodológicos}

La ontología idealista, da una explicación de este suceso, ya que un objeto físico u observable, no puede tener existencia en sí mismo, sino existe un ser con mente cognoscente que sea consciente de dicho objeto (Briones, 2002). Por ello, la construcción social y espacial de la realidad donde se embebe el individuo no existe si su mente y pensamiento no "aprehende" una situación, problema o fenómeno de interés particular o comunitario (Bunge, 2002).

Por lo tanto, la relación investigador-objeto de estudio es concomitante, ya que existe una participación democrática y comunicativa entre el investigador y los sujetos investigados (Colomer, 1990). En consecuencia, la construcción del conocimiento se da de manera colaborativa, armónica y dinámica, siendo la reflexión, el procedimiento metodológico utilizado por excelencia, que posibilita el análisis y tratamiento de las ideas y percepciones de la conciencia trascendental, ya sea a través de matrices de análisis de contenido, triangulaciones de investigadores y de métodos, permitiendo la contrastan las opiniones de los participantes del estudio, con el análisis de las impresiones registradas en instrumentos como: el diario de campo y registro anecdótico in situ, con el marco referencial teórico, en base a las categorías de análisis que emergieron durante la interacción entre el investigador y el auditorio.

Considerando a la entrevista y la observación sistemática, como las técnicas modelos la extracción y producción de conocimiento. Su lógica, es el conocimiento que permita al investigador entender lo que está pasando con su objeto de estudio, a partir de la interpretación ilustrada. Por consiguiente, no busca la verificación del conocimiento, solo el descubrimiento e interpretación del mismo, a partir de sus versionantes (González, 2007).

\subsection{Fundamentos teóricos-conceptuales distintivos de la investigación cualitativa}

La investigación cualitativa; es un tipo de investigación cuya finalidad es proporcionar una mayor comprensión, significados e interpretación subjetiva que el hombre da a sus creencias, motivaciones y actividades culturales, a través de diferentes diseños investigativos, ya sea a través de la etnografía, fenomenología, investigaciónacción, historias de vida y teoría fundamentada (Behar, 2008).

Entre las características más sobresalientes de la investigación cualitativa se pueden mencionar (Vasilachis et al., 2006):

1. No hay realidad objetiva, ya que la misma se construye socialmente a partir de los actores o informantes claves.

2. El conocimiento (episteme) emerge de las personas que participan activamente en la investigación.

Vivat Academia. Revista de Comunicación. 15 septiembre/15 diciembre, 2018, nº 144, 69-76 


\section{Corona Lisboa, J. L. Investigación cualitativa: fundamentos epistemológicos, teóricos y metodológicos}

3. La tarea primordial del investigador, es entender e interpretar el mundo de los participantes en base a sus experiencias y visiones del entorno donde se desenvuelven.

4. El investigador y los participantes interactúan de manera constante y dinámica durante todas las etapas de la investigación.

5. La investigación es un producto mosaico resultante de los valores culturales, étnicos y del investigador.

6. Es holística e integradora, porque no deja de lado cualquier información novedosa que surja durante la pesquisa. Por ello, los objetivos del estudio pueden cambiar en base a las vicisitudes durante la recogida de la información con los sujetos significantes (Muestra de estudio).

7. La investigación cualitativa no parte de supuestos verificables o hipótesis, ni de variables medibles cuantitativamente, ya que analiza información de carácter subjetiva que no es posible detectar a través de los sentidos y la inducción (afecto, valores culturales, motivaciones, entre otros).

Los puntos antes mencionados, deja de manifiesto que la investigación cualitativa se apoya en las observaciones naturalistas, basado en modelos comprensivos y comportamentales, con una óptica constructivista y transformadora de la realidad social, cualidades que solo se encuentran en esta metodología investigativa, permitiendo la emergencia de este enfoque sobre la visión positivista y neopositivista, dejando de lado la rigurosidad metodológica de las anteriores, para promover nuevas formas y matices que permitan a los investigadores adentrarse introspectivamente en las ideas y prácticas antropológicas distintivas de la especie humana, lo cual produjo una crisis dentro del paradigma cuantitativo, por no dar respuesta a aquellos patrones no medibles por fórmulas o procedimientos estadísticos y/o matemáticos, que son inalienables a la conducta humana como la afectividad, introspección, entre otros.

Por ello, se considera un enfoque novedoso y flexible, el cual está influenciado directamente por la cultura y las relaciones sociales particulares, que hacen que la realidad subjetiva dependa de la comprensión y análisis del conocimiento de las formas de percibir, pensar, sentir y actuar, del sujeto cognoscente.

\subsection{Filósofos y precursores de la investigación cualitativa}

El enfoque de investigación cualitativa ha recibido la influencia de importantes filósofos e investigadores como: Edgar Morín, Paulo Freire, Edmund Husserl, Martin Heidegger y Schütz Alfred, quienes aportaron una visión profunda de la interacción humana con el entorno subyacente y como los sujetos interpretan sus propias vivencias

Vivat Academia. Revista de Comunicación. 15 septiembre/15 diciembre, 2018, $\mathrm{n}^{\circ}$ 144, $69-76$ 


\section{Corona Lisboa, J. L. Investigación cualitativa: fundamentos epistemológicos, teóricos y metodológicos}

para adquirir conductas determinadas. En breve, se describen las contribuciones más significativas de estos precursores en el ámbito del enfoque interpretativo:

Edgar Morín: La visión compleja de la realidad crítica del hombre frente a su entorno, la plantea Morín (1999) en su teoría del "Pensamiento Complejo", quien parte de la necesidad imperante y urgente de la reconciliación de hombre con su entorno, junto a una mejora en las relaciones humanas, es decir, una metodología de pensamiento nuevo, para comprender la naturaleza, la sociedad, reorganizar la vida humana y para buscar soluciones a las crisis de la humanidad actual, siendo el capital humano la base intelectual del éxito organizacional.

Con ello, Morín expresa que en los procesos de generación y transmisión de conocimiento participan directamente seres humanos, con modelos mentales, conocimientos, valores, principios y creencias distintas, que influyen marcadamente en las percepciones, traducciones y reconstrucciones que él mismo hace sobre la información recibida.

Paulo Freire: Plantea que la educación y la investigación no es un acto de transmisión de conocimientos, si no que por el contrario se va formando mediante un mundo en común y que su adquisición es de forma continua, porque los seres humanos somos seres de transformación y no de adaptación, lo cual implica cultivar el conocimiento desde la propia realidad del hombre (auto-aprendizaje) sin un método riguroso que limite el accionar del sujeto en busca de la destrucción de las barreras de la ignorancia (García y Puigvert, 1998).

El postulado de Friere, conlleva a una reflexión intrínseca del yo interno en concordancia con la metodología biográfica y la investigación-acción, en busca de la transformación social continua y la emancipación de los pueblos oprimidos por falta de una cultura del conocimiento, es decir, el hombre no se hace en el silencio, sino en la palabra, la acción y la auto-retrospección.

Edmund Husserl: La visión de Husserl lleva a conocer parte de la vida de los participantes de la investigación, así como su propio mundo, relacionados con el fenómeno en estudio, es decir, su punto de partida es la vida de las personas, enfocándose en cómo las experiencias, significados, emociones y situaciones del individuo en estudio son percibidos, aprendidos y concebidos (Barbera e Inciarte, 2012; Lámbert, 2006).

Esta nueva perspectiva aporta una característica novedosa, ya que nos transporta hacia las puertas del diseño fenomenológico y a la esencia interpretativa de la hermenéutica, permitiendo estudiar e interpretar la necesidad de apropiación del significado que subyace en las experiencias vividas por el individuo en relación a su entorno físico y biológico, donde la verdad absoluta no existe, ya que todo depende de la subjetividad intrínseca del hombre.

Vivat Academia. Revista de Comunicación. 15 septiembre/15 diciembre, 2018, n $144,69-76$ 


\section{Corona Lisboa, J. L. Investigación cualitativa: fundamentos epistemológicos, teóricos y metodológicos}

Martin Heidegger: Este filósofo dio un matiz interesante a fenomenología social, ya que fundó las bases del conocimiento sobre la experiencia de la existencia propia, a lo que llamó "El Dasein", es decir, "el ser ahí" donde el hombre es el centro de su propia realidad y sólo él, puede crear sus ideas y prácticas sociales según el diario acontecer y el impacto que esas vivencias tienen en su toma de decisiones y visión del mundo (Buitrón y Calderón, 2012; Corona et al., 2016f).

Heidegger, pretende explicar que el hombre es el artífice de su propio destino y en consecuencia de su accionar en el mundo donde se desenvuelve. El ser cognoscente es el epicentro de la historia, por lo tanto, él construye su propia realidad según el vivir cotidiano y la interacción con otros semejantes, influyendo en la cimentación de patrones étnicos y culturales dentro de la sociedad.

Schütz Alfred: Se considera uno de los filósofos más importantes dentro de la investigación sociológica. Introdujo la metodología de Husserl para estudiar y comparar distintos grupos sociales, distinguiendo dos términos utilizados hasta la actualidad: actor y observador. En el primero hace referencia a los sujetos pertenecientes a un grupo o etnia y el segundo; al sujeto que indaga o investiga a los actores, para comprender e interpretar los significados que ellos dan a diversos objetos (Morales, 2011). No obstante, el actor puede cambiar su rol a observador y viceversa, algo similar a lo que sucede en las investigaciones con diseños IAP (investigación-acción-participante), donde existen interacciones recíprocas entre los sujetos significantes y el investigador.

Schütz complementó las ideas de Husserl y en cierto modo los planteamientos de Heidegger, ya que las relaciones entre los sujetos ocurren en un contexto sociocultural diverso y dinámico, nada es estático en la sociedad, las ocurrencias de fenómenos van y vienen, siendo la razón principal de las divergencias de culturas, religiones e ideas que impregnan la especie humana, en un mundo heterogéneo y subjetivo.

\section{CONCLUSIÓN}

La investigación cualitativa es un paradigma emergente que sustenta su visión epistemológica y metodológica en las experiencias subjetivas e intersubjetivas de los sujetos, cuya práctica se orienta hacia la sociedad construida por el hombre, donde interactúan las versiones y opiniones del ser pensante, respecto a los hechos y fenómenos de estudio, para construir la realidad de manera cooperativa y dinámica.

\section{BIBLIOGRAFÍA}

Barbera. N., y Inciarte, A. (2012). Fenomenología y hermenéutica: dos perspectivas para estudiar las ciencias sociales y humanas. Multiciencias, 12(2), 199-205.

Behar, D. (2008). Metodología de la investigación. México: Editorial Shalom Bunge, M. (2002). Ser, Saber, Hacer. México: Editorial Paidós

Briones, G. (2002). Epistemología de las ciencias sociales. Bogotá: ARFO Editores

Vivat Academia. Revista de Comunicación. 15 septiembre/15 diciembre, 2018, nº 144, $69-76$ 
Butrón, M., y Calderón, L. (2012). El Proceder Fenomenológico y Hermenéutico en la Construcción de Significados. Reflexionando una experiencia. Revista electrónica REDINE - UCLA, 2(7), 84-100.

Colomer, E. (1990). Historia del Pensamiento Alemán de Kan a Heidegger. Barcelona: Editorial Herder.

Corona, J. (2016a). Apuntes sobre métodos de investigación. Medisur, 14(1).

Corona, J. (2016b). Investigación científica. A manera de reflexión. Medisur, 14(3).

Corona, J., Kovac, M., Mijares, M., y Grimaldos, R. (2016). The phenomenology of Edmund Husserl, Martin Heidegger and Alfred Schütz. International Journal of Philosophy and Social-Psychological Sciences, 2(4), 86-90.

García, R., y Puigvert, L. (1998). Aportes de Paulo Freire a la Educación y las Ciencias Sociales. Revista Interuniversitaria de Formación del Profesorado, 33, 21-28.

González, A. (2007). Investigación cualitativa. La Habana: Editorial Ecimed.

Hernández, A., Padrón, J. (1997). Referencias Básicas en la Producción de una Tesis Doctoral. San Juan de los Morros: Colegio de Economistas

Lambert, C. (2006). Edmund Husserl: La idea de la fenomenología. Facultad Cs. Religiosas y Filosóficas. Universidad Católica del Maule. Teología y Vida, 47.

Morales, J. (2011). Fenomenología y hermenéutica como epistemología de la investigación. Revista Paradigma, 22(2), 7-22.

Morín, E. (1999). Pensamiento Complejo. París: Editorial L Harmattan.

Vasilachis, I., Ameigeiras, A., Chernobilsky, L., Giménez, V., Mallimaci, F., Mendizábal, N., Neiman, G.,Quaranta, G., y Soneira, A. (2006). Estrategias de investigación. México: Centro Panamericano de estudios Superiores.

\section{AUTOR}

\section{Jose Luis Corona Lisboa}

Licenciado en Educación, Mención: Biología. MSc. En Gestión y Auditorías Ambientales en Ciencia y tecnología Marina. MSc. En Producción Animal. Profesor e investigador de la Universidad Nacional Experimental Francisco de Miranda, Venezuela. https://scholar.google.es/citations?hl=es\&pli=1\&user=s5ouOFkAAAAI

Vivat Academia. Revista de Comunicación. 15 septiembre/15 diciembre, 2018, n $144,69-76$ 